patients undergoing targeted BRAF inhibitor treatment for metastatic melanoma. JAMA Dermatol 2014; 150: 307-311.

7 Bisschop C, Bruijn MS, Stenekes MW, Diercks GFH, Hospers GAP. Foreign body reaction triggered by cytotoxic $\mathrm{T}$ lymphocyte-associated protein 4 blockade 25 years after dermal filler injection. Br J Dermatol 2016; 175: 1351-1353.

8 Lee JM, Kim YJ. Foreign body granulomas after use of dermal fillers: pathophysiology, clinical and appearance, histologic features, and treatment. Arch Plast Surg 2015; 42: 232-239.

9 Korman AM, Nisar MS, Somach SC. Subclinical granulomas in benign skin lesions heralding the onset of BRAF and MEK inhibitor-associated granulomatous dermatitis in a patient with metastatic melanoma. JAAD Case Reports 2018; 4: 722-724.

10 Tetzlaff MT, Nelson KC, Diab A et al. Granulomatous/sarcoid-like lesions associated with checkpoint inhibitors: a marker of therapy response in a subset of melanoma patients. J Immunother Cancer 2018; 6: 14

11 Chen L, His AC, Kothari A et al. Granulomatous dermatitis secondary to vemurafenib in a child with Langerhans cell histiocytosis. Pediatr Dermatol 2018; 35: e402-e403.

DOI: $10.1111 / j d v .16550$

\section{Fusariosis manifesting as targetoid purpuric cutaneous lesions in immunocompromised patients}

\section{Editor,}

Fusarium is a geophilic fungus, widely distributed in soil all around the world. Pathogenic subtypes may cause opportunistic infections in humans, particularly in patients with underlying immunosuppression.

We have encountered disseminated fusariosis in three patients (case 1: a 46-year-old male; case 2: a 50-year-old female; case 3: a 33-year-old female), who had undergone allogeneic hematopoietic stem cell transplantation (HSCT). The infection developed 37, 60 and 75 days after HSCT, respectively. All patients had prolonged ( $>10$ days) and profound $\left(<100 / \mathrm{mm}^{3}\right)$ neutropenia and presented with persistent high fever refractory to broadspectrum antibiotics. Dermatologic examination revealed tender purpuric targetoid macules and plaques with central necrotic eschars. The cutaneous eruption displayed an abrupt onset and an accelerated course with eventual widespread dissemination to trunk, head and extremities (Figs 1 and 2c). Histopathological examination in all cases revealed fungal septated and branched hyphae and yeasts in the dermis between the collagen fibres and within the blood vessels. PAS stain highlighted the fungal elements (Fig. 2a). Fusarium spp were isolated from fungal culture on days 69,75 and 110 , respectively (Fig. $2 \mathrm{~b}$ ). After the diagnosis was firmly established as fusariosis, a combination of intravenous voriconazole (first day $6 \mathrm{mg} / \mathrm{kg}$ twice daily, then $4 \mathrm{mg} /$ $\mathrm{kg}$ twice daily) and liposomal amphotericin B ( $5 \mathrm{mg} / \mathrm{kg} / \mathrm{day})$ was introduced. Unfortunately, this therapy could not halt the progression of the infection and patients succumbed to disease at 72, 118 and 137 days after HSCT, respectively.

Fusariosis in the immunocompromised population is typically an invasive or disseminated infection with a poor outcome. In contrast to other fungal infections, fusarium stands out with its ability to cause fungemia. The mortality rate usually ranges from $60 \%$ to $80 \%$. Fusariosis is the second most common invasive mould infection in HSCT recipients. ${ }^{1,2}$ Figures on the incidence of invasive fusariosis in allogeneic HSCT recipients are varied: $0.2 \%$ in USA, ${ }^{3} 3 \%$ in Italy ${ }^{4}$ and $5.2 \%$ in Brazil. ${ }^{5}$ We have encountered four cases among 417 allogeneic HSCT recipients within 8 years; the incidence was $0.9 \%$ in our experience.

Among the numerous underlying risk factors for fusariosis, the principal ones are neutropenia and impaired T-cell function. All patients described herein had received calcineurin inhibitors and high-dose steroids (as a prophylaxis for graft versus host disease), which powerfully inhibit T-cell function. In addition, all of them had impaired neutrophil engraftment as the cause of their neutropenia, which is another risk factor for fusariosis.

The major portal of entry for Fusarium spp is the airways, followed by skin at sites of tissue breakdown, and possibly the mucous membranes. It has been disclosed that the hospital plumbing system may be a reservoir for Fusarium spp. ${ }^{6}$ All patients in this report had severe mucositis on admission, which might represent the portal of entry for the infection.

The typical clinical scenario for fusariosis comprises abrupt onset of painful purpuric macules, patches and plaques with central necrosis, in a severely neutropenic patient with high fever. Targetoid lesions may simulate ecthyma gangrenosum or purpura fulminans. We have also observed radial fresh purpuric globules around targetoid necrotic lesions (Fig. 1).

Accurate diagnosis of fusariosis may be accomplished through blood culture, skin biopsy, fungal culture, PCR and 1,3-ßeta-Dglucan antigen testing. Although fungal culture is a prerequisite for precise diagnosis of fusariosis, skin biopsy is the quickest and

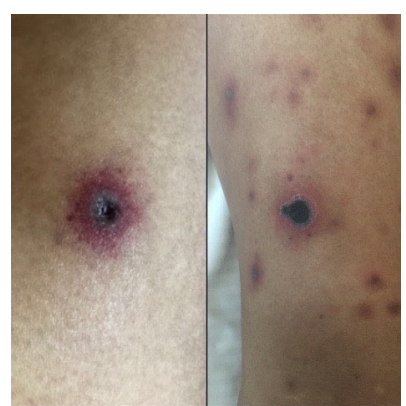

Figure 1 Ecthyma gangrenosum-like lesions were noted in two of our patients with fusariosis (Case 1 and case 3 ). 


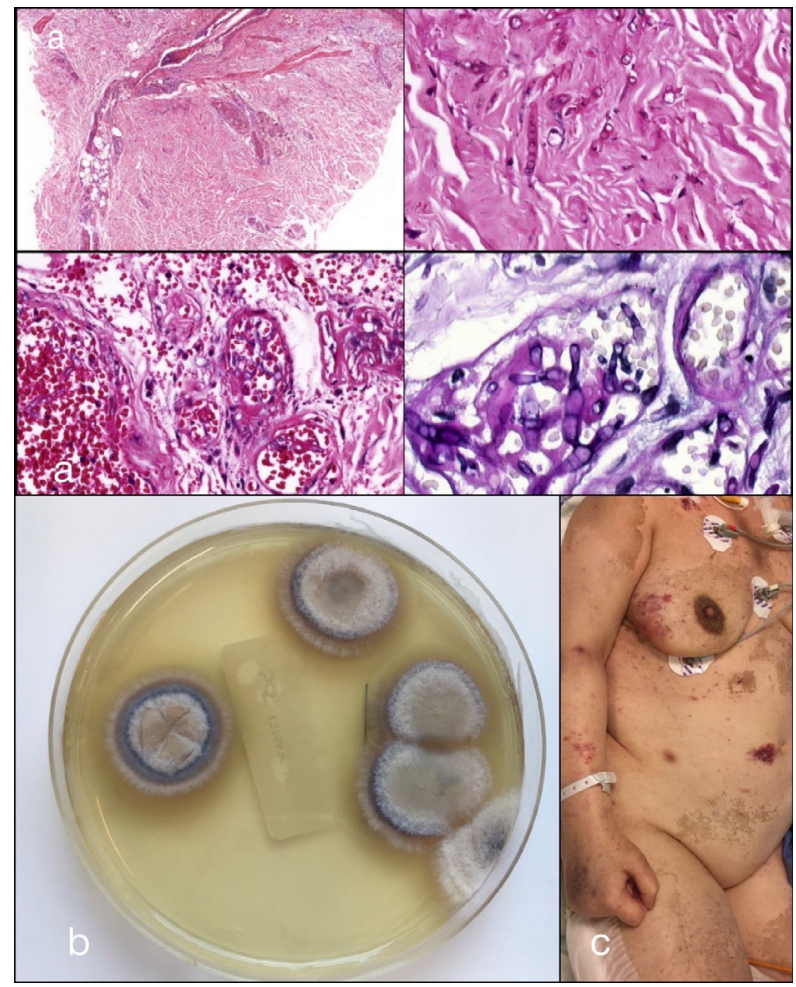

Figure 2 (a) Upper left $(H \& E \times 40)$ and upper right $(H \& E \times 200)$ : Fungal hyphae and yeasts in the dermis between the collagen fibres. Lower left $(\mathrm{H} \& \mathrm{E} \times 400)$ : Fungal septated and branched hyphae within the blood vessels. Lower right (PAS $\times 400)$ : PAS stain highlighting the fungal elements. (b) Fusarium spp grown in Sabouraud culture. (c) Clinical presentation of case 2.

easiest way for a presumptive diagnosis until the culture results are available. ${ }^{7}$

The optimal treatment for invasive fusariosis is unclear. Nucci et al. ${ }^{7}$ recommend either voriconazole or liposomal amphotericin B as primary therapy, despite occasional rewarding outcomes. The most important positive prognostic factor is resolution of neutropenia.

In conclusion; disseminated fusariosis should be considered in all patients presenting with high fever and widespread targetoid purpuric and necrotic cutaneous lesions, in the context of profound and protracted neutropenia and/or T-cell dysfunction.

\section{Acknowledgement}

The patients presented in this manuscript have given written informed consent to publication of their case details during biopsy procedure.

\section{Funding sources}

none.

$$
\begin{array}{r}
\text { Y. Erdemoglu, }{ }^{1,2} \text { (DD I.E. Aydingoz, }{ }^{1,2} \text { C. Ustun, }{ }^{3} \text { A. Uzay, },{ }^{1,4} \\
\text { Y. Besli, }{ }^{1,5} \text { E. Ozturk Durmaz, }{ }^{1,2,{ }^{*}} \text { C. Demirkesen, }{ }^{1,6} \\
\text { S. Sahin }{ }^{1,2}
\end{array}
$$

${ }^{1}$ Acibadem Mehmet Ali Aydınlar University School of Medicine, Istanbul, Turkey, ${ }^{2}$ Department of Dermatology, Acibadem University, Istanbul, Turkey, ${ }^{3}$ Department of Infectious Diseases, Hasan Kalyoncu University, Istanbul, Turkey, ${ }^{4}$ Department of Hematology, Acibadem University, Istanbul, Turkey, ${ }^{5}$ Department of Microbiology, Acıbadem University, Istanbul, Turkey, ${ }^{6}$ Department of Pathology, Acibadem University, Istanbul, Turkey

*Correspondence: E. Ozturk Durmaz. E-mail: emelerkek@yahoo.com

\section{References}

1 Kontoyiannis DP, Marr KA, Park BJ et al. Prospective surveillance for invasive fungal infections in hematopoietic stem cell transplant recipients, 2001-2006: overview of the Transplant-Associated Infection Surveillance Network (TRANSNET) database. Clin Infect Dis 2010; 50: 1091-1100.

2 Pagano L, Caira M, Nosari A et al. Fungal infections in recipients of hematopoietic stem cell transplants: results of the SEIFEM B-2004 studySorveglianza Epidemiologica Infezioni Fungine Nelle Emopatie Maligne. Clin Infect Dis 2007; 45: 1161-1170.

3 Nucci M, Garnica M, Gloria $\mathrm{AB}$ et al. Invasive fungal diseases in haematopoietic cell transplant recipients and in patients with acute myeloid leukaemia or myelodysplasia in Brazil. Clin Microbiol Infect 2013; 19: 745-751.

4 Marr KA, Carter RA, Crippa F, Wald A, Corey L. Epidemiology and outcome of mould infections in hematopoietic stem cell transplant recipients. Clin Infect Dis 2002; 34: 909-917.

5 Anaissie EJ, Kuchar RT, Rex JH et al. Fusariosis associated with pathogenic Fusarium species colonization of a hospital water system: a new paradigm 
for the epidemiology of opportunistic mold infections. Clin Infect Dis 2001; 33: 1871-1878.

6 Girmenia C, Arcese W, Micozzi A, Martino P, Bianco P, Morace G. Onychomycosis as a possible origin of disseminated Fusariumsolani infection in a patient with severe aplastic anemia. Clin Infect Dis 1992; 14: 1167.

7 Nucci F, Nouér SA, Capone D, Anaissie E, Nucci M. Fusariosis. Semin Respir Crit Care Med 2015; 36: 706-714.

DOI: $10.1111 / j d v .16551$

\section{Effectiveness and adverse events of ivermectin treatment for scabies in $\mathbf{3 0}$ infant patients: report from a German single centre}

\section{Dear Editor,}

Scabies is a globally prevalent parasitic skin infestation characterized by severe pruritus and a heterogeneous clinical picture depending on the immune status of the individual. Epidemiologic studies reveal a higher susceptibility for young, old and, in general, immunocompromised individuals. Disease-related morbidity and secondary complications due to a disrupted epidermal barrier, e.g. subsequent bacterial infections and chronic kidney disease, further add to the patients' burden. ${ }^{1}$ With current treatment options, induction of remission is achieved in most of the patients. Specifically, the oral administration of ivermectin has substantially decreased the prevalence of scabies in communities and has proven to be effective and tolerable. ${ }^{2,3}$ However, relapses in larger families are frequent and ivermectin is not approved for children $<15 \mathrm{~kg}$. Hence, evaluation of the safety of ivermectin use in infants is highly warranted. Recently, a multicentric observational study has reported on the safety and efficacy of ivermectin use in infants weighing $<15 \mathrm{~kg}^{4} .85 \%$ of 170 patients achieved remission and in $4 \%$ of the patients, mild adverse effects were observed. However, safety of ivermectin remains controversial, ${ }^{5,6}$ and controlled studies in infants are scant. ${ }^{7,8}$ We therefore retrospectively analysed 30 infants $<15 \mathrm{~kg}$ infected with scabies. The patients were treated off-label with oral ivermectin under controlled and standardized conditions during hospitalization between 01/2018 and 01/2020. The focus of our observation was on safety and efficacy. This study was approved by the local ethics committee.

After informed consent by the legal guardian(s), 30 patients [age (months), mean $\pm \mathrm{SD}: 12 \pm 9.2$; weight $(\mathrm{kg})$, mean \pm SD: $8.6 \pm 2.5,16$ males, 14 females] were treated with oral ivermectin after clinical and dermatoscopic diagnosis of scabies. All patients had been unsuccessfully treated with topical permethrin at least twice prior to ivermectin administration and were at least 2 months of age. Prior topical treatment with permethrin creme $5 \%$ was applied overnight on the entire body, including the head and neck, at days 0 and 7. Concomitantly, all individuals with close physical contact to the scabies-infected patient received appropriate scabies treatment. Supportively, recommended hygienic measures were applied. A relapse was defined as a recurrent clinical skin manifestation caused by scabies mites as detected by dermatoscopy 4 weeks after treatment. Reasons for relapses in these patients remain speculative; however, they might be explained either by (i) a lack of efficacy of the topical permethrin treatment or by (ii) the insufficient application in an outpatient setting. At days 0 and 7 , ivermectin capsules were orally applied at a dose of $200 \mu \mathrm{g}$ per $\mathrm{kg}$ bodyweight; concomitantly, topical permethrin $5 \%$ treatment was applied. To monitor safety, all patients were hospitalized for at least 2 days and blood samples were obtained, in total for 4 times, specifically, before and $24 \mathrm{~h}$ after ivermectin administration both at days 0 and 7. To determine ivermectin-related pathological changes, findings in blood samples collected before ivermectin treatment were used as a reference and were compared to results in blood samples obtained after ivermectin treatment. In most cases, final clinical and dermatoscopic examination was performed at a follow-up visit on day 14 .

After treatment, 4 patients (2/4 at day 1 and $2 / 4$ at day 8 ) revealed slightly elevated creatine kinase levels but no further pathological findings in the routine laboratory examination. No severe-grade $2-5$ adverse events were observed. A total of 21 patients showed no detectable mites and regredient inflammatory skin lesions. Four additional patients did not return to our clinic but were contacted by telephone and confirmed clinical remission. In 5 patients, we lost follow-up.

While our study provides first insights into the safety and efficacy of oral ivermectin in infants in Germany, the findings need to be interpreted within the limitations of the study, specifically, (i) the retrospective study design, (ii) the focus on a single centre and (iii) the limited sample size. In addition to the questionnaire-based multicentre study on 170 patients performed by Levy et al., ${ }^{4}$ we here provide a safety monitoring under controlled inpatient conditions and based on routine blood examination.

Despite the limitations of our study, our observations suggest the safe and effective use of ivermectin in infants $<15 \mathrm{~kg}$ infected with scabies and warrant the initiation of a prospective controlled study for this indication.

\section{Funding sources}

The study was supported by the Excellence Cluster 'Precision Medicine in Chronic Inflammation' (EXC 2167/1) and the Research Training Group 'Modulation of Autoimmunity' (GRK 1727/2) both from the Deutsche Forschungsgemeinschaft. 